\title{
INEQUALITIES FOR GAUSSIAN HYPERGEOMETRIC FUNCTIONS
}

\section{XIAO-YAN MA* AND TI-REN HUANG}

Abstract. In this paper, the authors present several hypergeometric transformation inequalities for the Gaussian hypergeometric function $F(a, b ; c ; x)$, which are the extensions of the known hypergeometric transformation identities such as Ramanujan's cubic transformation identities, by showing the monotonicity properties of certain quotients of $F(a, b ; c ; x)$ and its special cases. By these results, some related known results are considerably improved. Mathematics subject classification (2010): 33C05, 39B62.

Keywords and phrases: Gaussian hypergeometric function, Ramanujan's transformation, infinite product.

\section{REFERENCES}

[1] G. Almkvist, B. Berndt, Gauss, Landen, Ramanujan, the arithmetic- geometric mean, ellipses, $\pi$, and the Ladies Diary, American Mathematical Monthly, 95, 7 (1988), 585-608.

[2] Á. BARICZ, Landen-type inequality for Bessel function, Computational Methods and Function Theory, 5, 2 (2005), 373-379.

[3] Á. BARICZ, Generalized Bessel functions of the first kind. Lecture Notes in Mathematics 1994, Springer-verlag, Berlin Heidelberg, 2010.

[4] Á. BARICZ, Landen Inequalities for special functions, Proceeding of the American mathematical society, 142 (2014), 3059-3066.

[5] B. C. Berndt, S. Bhargava, F. G. Garvan, Ramanujan's theories of elliptic functions to alternative bases, Transactions of the American Mathematical Society, 347, 11 (1995), 4163-4244.

[6] J. M. Borwein, P. B. BorweIn, Pi and the AGM: A study in analytic number theory and computational complexity, John Wiley \& Sons, New York, 1987.

[7] F. G. GAVRVAN, Ramanujan's theories of elliptic functions to alterative bases-a symbolic excursion, J. Symbolic computation, 20 (1995), 517-536.

[8] X. Y. MA, S. L. QIU, G. Y. TU, Generalized Grötzsch ring function and generalized elliptic integrals, Applied Mathematics-A Journal of Chinese Universities. Series B. 31, 4 (2016), 458-468.

[9] S. Ponnus Amy, M. VuORInen, Asymptotic expansions and inequalities for hypergeometric functions, Mathematika, 44, 2 (1997), 278-301.

[10] S. L. QIU, M. Vuorinen, Special functions in geometric function theory. In book: Handbook of Complex Analysis: Geometric Function Theory, Elsevier Sci B V, Amsterdam, 2 (2005), 621-659.

[11] S. TAKEUCHI, A new form of the generalized complete elliptic integrals, Kodai Mathematical Journal, 39, 1 (2016), 202-226.

[12] S. TAKEUCHI, The complete p-elliptic integrals and a computation formula of $\pi_{p}$ for $p=4$, The Ramanujan Journal, 46 (2018), 309-321.

[13] M. K. WANG, Y. M. CHU, Y. P. JIANG, Ramanujan's cubic transformation and generalized modular equation, http://arxiv.org/pdf/1305.6525.pdf.

[14] M. K. WANG, Y. M. CHU, Y. P. JIANG, Ramanujan's cubic transformation and inequalities for zerobalanced hypergeometric functions, Rocky Mountain Journal of Mathematics, 46, 2 (2016), 679-691.

[15] M. K. WANG, Y. M. CHU, Y. P. JIANG, D. D. YAN, A class of quadtatic transformation inequations for zero-balanced hypergeometric functions, Acta Mathematica Scientia, 34 A,4 (2014), 999-1007.

[16] W. WANG, J. J. PAN, S. G. YANG, Inequalities for Gaussian hypergeometric functions, Journal of Mathematical Inequalities, 11, 1 (2017), 27-39. 